\title{
Development of RNA-Based Assay for Rapid Detection of SARS-CoV-2 in Clinical Samples
}

\author{
Vinod Kumar ${ }^{a}$ Suman Mishra ${ }^{a}$ Rajni Sharma ${ }^{a}$ Jyotsna Agarwal ${ }^{b}$ \\ Ujjala Ghoshal ${ }^{c}$ Tripti Khannad ${ }^{d}$ Lokendra K. Sharma $^{a}$ Santosh Kumar Verma ${ }^{a}$ \\ Prabhakar Mishra ${ }^{\mathrm{e}}$ Swasti Tiwaria \\ aDepartment of Molecular Medicine \& Biotechnology, Sanjay Gandhi PGIMS, Lucknow, India; bepartment of \\ Microbiology, Dr. Ram Manohar Lohia Institute of Medical Sciences, Lucknow, India; 'Department of Microbiology, \\ Virology and COVID19 Lab, Sanjay Gandhi PGIMS, Lucknow, India; Indian Council of Medical Research, New Delhi, \\ India; ' Department of Biostatistics and Health Informatics, Sanjay Gandhi PGIMS, Lucknow, India
}

\section{Keywords}

Coronavirus disease-19 - Diagnosis · Ribonucleic acid ·

Coronavirus disease $\cdot$ Colorimetric test

\begin{abstract}
Introduction: The ongoing spread of pandemic coronavirus disease-19 (COVID-19) caused by severe acute respiratory syndrome coronavirus 2 (SARS-CoV-2) is of growing concern. Rapid diagnosis and management of SARS-CoV-2 are crucial for controlling the outbreak in the community. Here, we report the development of a first rapid-colorimetric assay capable of detecting SARS-CoV-2 in the human nasopharyngeal RNA sample in less than 30 min. Method: We utilized a nanomaterial-based optical sensing platform to detect RNAdependent RNA polymerase gene of SARS-CoV-2, where the formation of oligo probe-target hybrid led to salt-induced aggregation and change in gold-colloid color from pink to blue visibility range. Accordingly, we found a change in colloid color from pink to blue in assay containing nasopharyngeal RNA sample from the subject with clinically diagnosed COVID-19. The colloid retained pink color when the test in-
\end{abstract}

Karger@karger.com www.karger.com/int

Karger $\stackrel{\text { ' }}{5}$

GOPEN ACCESS
(C) 2022 The Author(s)

Published by S. Karger AG, Basel

This is an Open Access article licensed under the Creative Commons Attribution-NonCommercial-4.0 International License (CC BY-NC) (http://www.karger.com/Services/OpenAccessLicense), applicable to the online version of the article only. Usage and distribution for commercial purposes requires written permission. cludes samples from COVID-19 negative subjects or human papillomavirus-infected women. Results: The results were validated using nasopharyngeal RNA samples from positive COVID-19 subjects $(n=136)$. Using real-time polymerase chain reaction as gold standard, the assay was found to have $85.29 \%$ sensitivity and $94.12 \%$ specificity. The optimized method has detection limit as little as $0.5 \mathrm{ng}$ of SARS-CoV-2 RNA. Conclusion: We found that the developed assay rapidly detects SARS-CoV-2 RNA in clinical samples in a costeffective manner and would be useful in pandemic management by facilitating mass screening.

(c) 2022 The Author(s).

Published by S. Karger AG, Basel

\section{Introduction}

Coronavirus disease has rapidly spread across the world raising severe global health concerns. In December 2019, China reported the first disease case in its Hubei Province. Based on the phylogenetic analysis, the identi-

Vinod Kumar, Suman Mishra, and Rajni Sharma equal authorship.
Correspondence to:

Swasti Tiwari, tiwaris@sgpgi.ac.in 
fied novel coronavirus has been named as severe acute respiratory syndrome coronavirus 2 (SARS-CoV-2), and the disease spread by SARS-CoV-2 became known as "COVID-19" being declared for a pandemic by World Health Organization (WHO) [1]. Despite global massive efforts to control the outbreak of COVID-19, this pandemic is still rising and developing an affordable screening for rapid diagnosis is critically required in the management of COVID-19 [2]. According to the WHO, the immediate priority is the development of point-of-care tests for the detection of SARS-CoV-2 at an early stage with improved sensitivity [3].

Currently, the COVID-19 diagnostic tests fall into two categories: antibody and nucleic acid-based detection systems. The developed immunoassay is rapid but inefficient for the detection of the pathogen at an early stage of infection [2]. Besides, specific situations are described by the WHO where antigen testing can be done [4]. Among the nucleic acid-based detection systems, the CDC considered a real-time polymerase chain reaction (RT-PCR)-based method as a gold standard for COVID-19 testing [5]. The sample-to-result time of the quantitative RT-PCR was initially $>4 \mathrm{~h}$; however, constant efforts are underway to improve the turn-around time through automation. Besides, time-consuming process PCR based tests are expensive; they require sophisticated instruments and expertise [2]. Thus, other technologies, such as reverse transcription-loop-mediated isothermal amplification [6], and Genome-editing [7], are being explored. These are promising technologies; however, the expected turn-around time would still be around $1 \mathrm{~h}$ and may not be economical for mass screening, especially for the developing countries.

Nanomaterials-based sensing platforms hold promise to develop rapid diagnostics. However, their limited use in the clinical setting is due to the need for sophisticated equipment $[8,9]$. A recent attempt to use antisense-nucleotide capped gold nanoparticles (AuNPs) for N-gene based, COVID-19 detection could be a game changer [10]. Whereas, WHO suggested that $\mathrm{N}$-gene has a relatively weak analytical capability as compared to the RNAdependent RNA polymerase (RdRp) gene, to detect COVID-19 infection [11]. Moreover, the authors have demonstrated COVID-19 detection in a cellular-model system, and application in human samples (in large sample number) has yet to be shown.

In this study, we report for the first time AuNPs-based rapid colorimetric assay for visual eye detection of COVID-19 RNA in human samples designed to target $R d R p$ specific gene target in very cost-effective manner with wide application of mass screening in field. We utilized the surface plasmon resonance property of AuNPs to detect unamplified COVID-19 RNA in human samples. The ability of AuNPs to preferentially adsorb ssRNA/ssDNA over dsDNA/dsRNA is the crucial concept of this assay. The single- and double-stranded oligonucleotides have different electrostatic properties, which provide stability (via retaining natural color) and aggregation (which causes color change) of AuNPs in solution, respectively [12].

\section{Materials and Methods}

\section{Chemicals}

Citrate buffer stabilized AuNPs (10 nm diameter) was purchased from Alfa Aesar (Thermo Fisher Scientific India Private Limited). Phosphate buffer saline ( $\mathrm{pH} 7.0$ ) and $\mathrm{NaCl}$ were procured from Sigma Aldrich. RdRp specific oligo probe sequence was adapted from the reference [11] and synthesized from Applied Biosystems. The sequence was approved by WHO and has reported specificity for coronavirus (SARS-CoV-2) only without any cross-reactivity with other respiratory viruses [11]. Flowchart of the methodology is described in Figure 1a.

\section{Clinical Samples}

For our study, we have not enrolled any participants; we received the left-over clinical samples (nasopharyngeal RNA samples) from the Department of Microbiology, SGPGIMS and RMLIMS, Lucknow. RNA extracted from the nasopharyngeal samples of person having symptoms like fever, cough, cold, sore throat, tiredness, aches, and pains were used in our study. Samples from patients below 18 years or pregnant women were not included in the study. The study protocol to use clinical samples was approved by Institutional Human Ethics Committee SGPGIMS, Lucknow (Ref N. PGI/BE/327/2020).

Nasopharyngeal RNA sample from subjects suspected to have COVID-19 infection ( $n=136)$ was used. The nasopharyngeal samples of the patients having symptoms were collected, and RNA was isolated in the Department of Microbiology, SGPGIMS, and RMLIMS, Lucknow. The nasopharyngeal swabs were collected in viral transport medium (Himedia, Cat No. AL167), and RNA was extracted by QIAamp Viral RNA Mini Kit (Qiagen, Cat. No. 52904) following manufacturer's instructions. The COVID-19 testing of these subjects was done in the Indian Council of Medical Research (ICMR, India) approved diagnostic laboratory using Taqmanbased RT-PCR kit (Labgun, lab, Genomics Co. Ltd, Republic of Korea).

The cervical DNA samples from human papillomavirus (HPV) infected woman $(n=2)$ was used as nonspecific target control. The clinical samples (cervical smear) for the HPV DNA test were processed using HPV Test Hybrid Capture ${ }^{\circledR} 2$ protocol (Qiagen). Samples with relative light units/cutoff value ratios $>1.0$ were considered as HPV positive and $<1.0$ were considered as HPV negative. Only HPV positive DNA samples were used during validation experiments. 
a

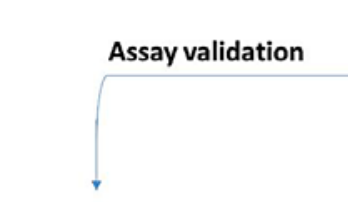

IVT SARS-CoV-2 RNA \&

Clinical samples b
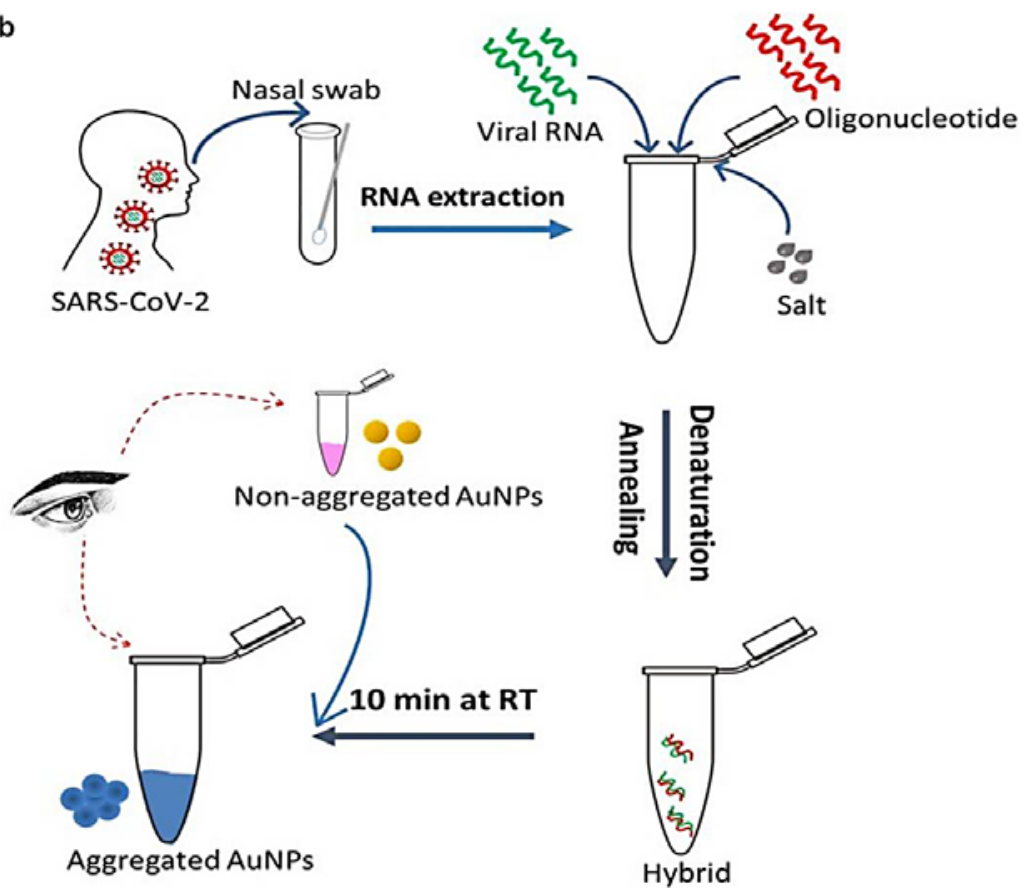

Assay sensitivity

Assay specificity

HPV DNA sample
IVT SARS-CoV-2 RNA \& synthetic SARS-CoV-2 control

Fig. 1. Flowchart of the methodology (a), schematic representation of the assay for the visual detection of SARSCoV-2 RNA (b). Schematic illustrates the assay flow to detect RdRp gene sequence of SARS-CoV-2 in the nasopharyngeal RNA sample from subject clinically diagnosed with COVID-19 (positive control).

In vitro Transcription of SARS-CoV-2 RNA

The RNA (5 ng) from confirmed COVID-19 positive human sample were first reverse transcribed with modified oligo dT primer having a T7 promoter sequence at its $5^{\prime}$ end, and the resulting single-stranded cDNA was further in vitro transcribed (IVT) using HiScribe T7 Quick High Yield RNA Synthesis Kit according to the manufacturer's protocol (New England BioLabs Inc., NEB \#E2050). The amplified RNA was than purified using Monarch RNA Cleanup Kit (New England BioLabs Inc.) and quantified by RNA HS reagent using Qubit system (Thermo Fisher Scientific).

Colorimetric Assay for the Detection of SARS-CoV-2 RNA

For colorimetric assay, reaction was set up in $10 \mu \mathrm{L}$ of reaction volume in sterile PCR tubes, containing (a) hybridization buffer containing $80 \mathrm{mM} \mathrm{NaCl}$, (b) $0.5 \mu \mathrm{M}$ oligo probe for $R d R p$ genes, and (c) target RNA from positive patients or IVT RNA. The RdRp oligo probe ( $5^{\prime}$-GTGATATGGTCATGTGTGGCGG-3') was used to specifically detect the presence of SARS-CoV-2 RNA in the assay. In parallel, to measure the specificity of the assay, input genomes from different sources such nasopharyngeal RNA from COVID-19 negative subjects and HPV DNA from cervical cancer positive samples were used as negative controls. Similarly, nontemplate control (NTC) was also included to measure the background reactivity. In addition, to confirm the working principle of the assay, a different RNA template (isolated from pancreas) and pancreas-specific regenerating islet-derived protein 3 (REG-3) gene oligo probe ( $5^{\prime}$ - GTGCCTATGGCTCCTATTGCT- $\left.3^{\prime}\right)$ were used separately.

The final reaction mixture with above combinations was then denatured at $95^{\circ} \mathrm{C}$ for $30 \mathrm{~s}$, annealed at $60^{\circ} \mathrm{C}$ for $60 \mathrm{~s}$ and then 
Table 1. Comparison of new color test by comparison to RT-PCR $(N=136)$

\begin{tabular}{llll}
\hline & \multicolumn{2}{l}{ RT-PCR (gold standard method) } & \multirow{2}{*}{ Total } \\
\cline { 2 - 3 } & positive & negative & \\
\hline New color test & & & \\
Positive & 58 & 4 & $\mathbf{6 2}$ \\
$\quad$ Negative & 10 & 64 & $\mathbf{7 4}$ \\
Total & $\mathbf{6 8}$ & $\mathbf{6 8}$ & $\mathbf{1 3 6}$ \\
\hline
\end{tabular}

cooled to room temperature for $10 \mathrm{~min}$. Subsequently, $10 \mathrm{nM}$ colloidal AuNPs $(\sim 10 \mathrm{~nm})$ were added to the assay mixture and allowed to develop color for 1-2 min.

\section{Spectral Studies and Measurement of Sensitivity}

Absorption spectrum of the assay mixture was recorded in the range of 300-700 nm. The peak shift from $520 \mathrm{~nm}$ (known as redshift) and peak broadening after $520 \mathrm{~nm}$ were measured as a characteristic feature of the salt-induced aggregation. Using various combinations of positive and negative controls (as discussed in the previous section), the specificity of reaction and aggregation were compared. Assay sensitivity was determined by serially diluting the input SARS-CoV-2 RNA from both the IVT synthesized and synthetic SARS-CoV-2 control (COVID-19 control kit, Cat. No. A47532, Applied Biosystems) ranging from 5 to $0.1 \mathrm{ng}$ and $1-0.1$ ng concentrations, respectively.

\section{Statistical Analysis}

Diagnostic accuracy of the new color test was calculated by assuming RT-PCR as gold standard method. Sensitivity (true positive rate), specificity (true negative rate), and overall accuracy (true positive and true negative rate) were calculated with $95 \%$ confidence interval. Likelihood ratio positive (sensitivity/false positive rate), likelihood ratio negative (false negative rate/specificity), positive predictive value (true positive value/total positive results predicted by color test), and negative predictive value (true negative value/total negative results predicted by color test) were also calculated. Measured accuracy was considered statistically significant $(p<0.05)$ when $50 \%$ did not falling within the lower and upper confidence limit of the confidence interval (expressed in \%). Statistical analysis was performed using software MedCalc for Windows (MedCalc Software, Ostend, Belgium).

\section{Results and Discussion}

In this study, we report for the first time the development of a rapid and affordable RNA-based assay for the visual detection of the SARS-CoV-2 genome in human samples. Using RT-PCR as gold standard, the developed assay was found to have a sensitivity of $85.29 \%$ and specificity of $94.12 \%$. For the assay, we use surface plasmon resonance property of AuNPs/colloids and targeted the

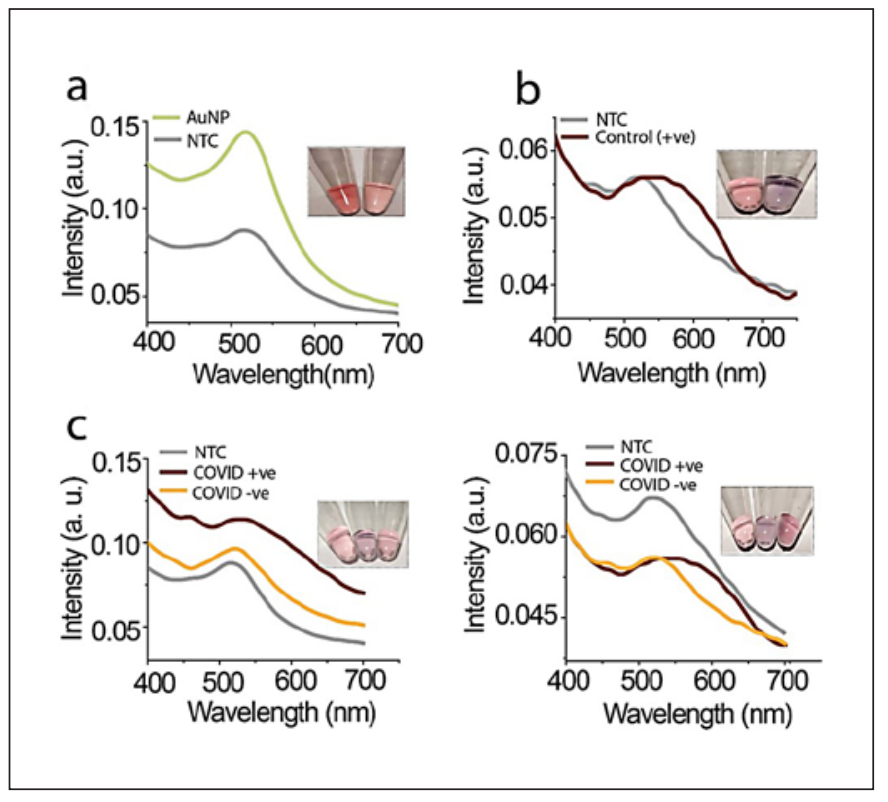

Fig. 2. Colorimetric assay to detect SARS-CoV-2 RNA. a Comparative absorption spectra of unmodified AuNPs (Green curve) and oligo probe stabilized AuNPs, i.e., NTC (Grey curve), optical images of gold colloids (left vial) and NTC (right vial) are shown in the inset. b Comparative absorption spectra of NTC (Grey curve) and positive control, i.e., nasopharyngeal RNA sample from subject clinically diagnosed with COVID-19 (Brown curve). Optical images shown in inset demonstrate the evident change in the color of the solution from pink to blue in the control vial (right) while no change in color of NTC vial (left). c Representative absorption spectra, and in the inset shows optical images comparing assay performed with NTC (Grey curves, left vial), RNA from clinically diagnosed COVID-19 subjects (Brown curve, middle vial), and RNA from subjects without COVID-19 (Yellow curve, right vial). Samples from a total of eighteen infected and eighteen uninfected individuals were analyzed (optical images were attached as online suppl. Fig. S2).

$R d R p$ specific gene sequence of SARS-CoV-2. $R d R p$ is essential for viral replication and has higher analytical power than $\mathrm{E}$ (envelope protein) and $\mathrm{N}$ (nucleocapsid protein) genes of SARS-CoV-2 (10). With the current escalated demand of cost effective, easy and sensitive diagnostic for COVID-19, the test was developed using commercially available SARS-CoV-2 synthetic DNA and validated further using clinical samples from COVID-19 subjects (as confirmed using Taqman-based RT-PCR method, online suppl. Table S1; for all online suppl. material, see www.karger.com/doi/10.1159/000522337). In the study, out of 136 samples, $50 \%$ samples $(n=68)$ were true positive (COVID-19 positive) and $50 \%$ samples $(n=$ 68) true negative (free from COVID-19 disease) confirmed by gold standard diagnostic test RT-PCR. These 
Table 2. Diagnostic accuracy of new color test by comparison to RT-PCR $(N=136)$

\begin{tabular}{llllll}
\hline Measurements & Value & \multicolumn{2}{l}{$95 \% \mathrm{Cl}$} & \multirow{2}{*}{$p$ value } \\
& & & lower & upper & \\
\hline Sensitivity (true positive rate), \% & 85.29 & 74.61 & 92.72 & $<0.05$ \\
Specificity (true negative rate), \% & 94.12 & 85.62 & 98.37 & $<0.05$ \\
LR+ & 14.50 & 5.58 & 37.71 & $<0.05$ \\
LR- & 0.16 & 0.09 & 0.28 & $<0.05$ \\
PPV, \% & 93.55 & 84.79 & 97.42 & $<0.05$ \\
NPV, \% & 86.49 & 78.26 & 91.92 & $<0.05$ \\
Overall accuracy, \% & 89.71 & 83.33 & 94.26 & $<0.05$ \\
\hline
\end{tabular}

Above estimation are based on equal number of disease- and disease-free cases in study samples detected by gold standard method. NPV, negative predictive value; PPV, positive predictive value; LR-, Likelihood ratio negative; LR+, Likelihood ratio positive true positive and negative samples were again tested by new color test to assess the diagnostic accuracy of our new test (Table 1). Result showed that sensitivity (true positive rate) of the new test was $85.29 \%$ (58/68) and specificity (true negative rate) of $94.12 \%(64 / 68)$ with statistically significant $p$ value $<0.05$ each. Overall diagnostic accuracy of the new color test was $89.71 \%(122 / 136)$ with statistically significant $p$ value $<0.05$. Likelihood ratio positive of our test is 14.50 , and likelihood ratio negative is 0.16 at significant $p$ value $<0.05$. Positive predicted value of $93.55 \%$ showed that probability to the correct prediction of true positive results from its own positive predicted results would be $93.55 \%$ and negative predictive value of $86.49 \%$ showed that probability to the correct prediction of true negative results from its own negative predicted results would be $86.49 \%$ (each $p<0.05$ ) (Table 2).

Figure $1 \mathrm{~b}$ illustrates the sequential schematics of process of the developed test. The color of the gold colloid solution is dependent on the aggregation property of AuNPs in suspension [13, 14]. Generally, in aqueous solution, gold colloids remain stabilized by the coating of negatively charged citrate ions [15] and have visible appearance of pink color (Fig. 1b). The same phenomenon was observed in our experiments when $R d R p$ oligo probe adsorbed and protected the salt-induced aggregation of colloid in the absence of target RNA (NTC) (Fig. 2a, Grey curve). Except for the reduced intensity of the absorption spectrum, which was due to dilution of the colloid (Fig. 2a, Grey curve), NTC and AuNP assays showed no change. Unlike dsDNA, inherent structural flexibility of ssDNA/ RNA to partially uncoil its bases, exposing them to AuNPs, generates the attractive electrostatic forces causing them to allow over colloids and giving protection against electrostatic interaction causing salt-induced ag-

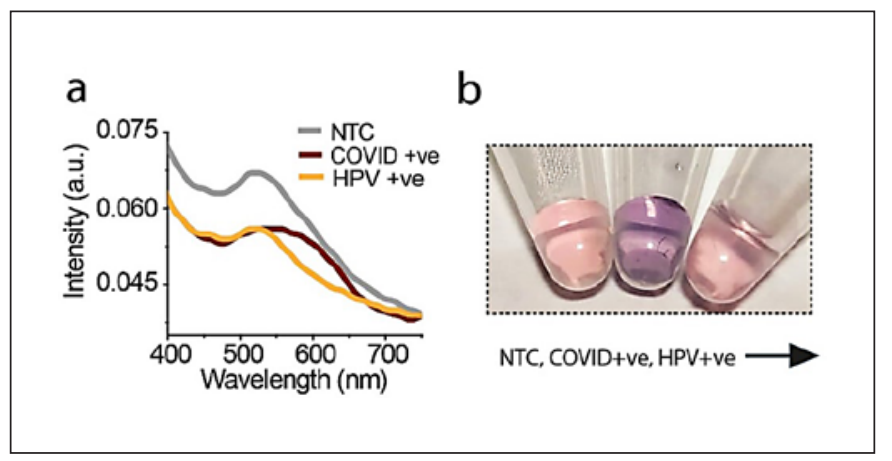

Fig. 3. Specificity of developed assay to detect SARS-CoV-2 RNA. Nasopharyngeal-RNA from COVID-19 subject (positive control), and a cervical DNA sample from HPV (nonspecific target control) infected women were tested. a Comparative absorption spectra for no target control (NTC, Grey curve), positive control (Brown curve), and nonspecific target control (HPV, Yellow curve). b Optical pictures demonstrate that the colloid color from pink to blue changes only in the vial with the positive control (middle vial), while the color remained pink in the vial with NTC (left vial) or HPV (right vial).

gregation [16]. In this assay, the colloid color changes in visible range from pink to blue indicate the formation of hybridized product (Fig. 2b, c, 3). Broadening of the peak with red-shift $(\sim 30 \mathrm{~nm})$ was observed in the spectrum of aggregated colloids than non-aggregated, confirms the success of developed assay for detection of an unamplified target with unmodified colloids in a quick and facile way. The principle of binding oligo probe was independently verified using a different template RNA (isolated from pancreas tissue) and pancreas-specific gene REG3 oligo probe in a separate assay. This assay also resulted in a similar change in color and absorption spectra as opti- 
Fig. 4. Sensitivity of developed assay to detect SARS-CoV-2 RNA. Assay sensitivity was determined using different concentrations (concentration are in ng) of IVT synthesized SARS-CoV-2 RNA (a, b), or synthetic DNA of SARS-CoV-2 (c, d). Absorption spectra $(\mathbf{a}, \mathbf{c})$ corresponding to aggregated colloids exhibit a clear red-shift in peak with broadening, indicating successful hybridization. Optical pictures (b, d) of the assay performed to demonstrate the color change, an extra pair of vials (on the left) to show color change with a higher amount (10 ng) of target nucleic acid for the reference.

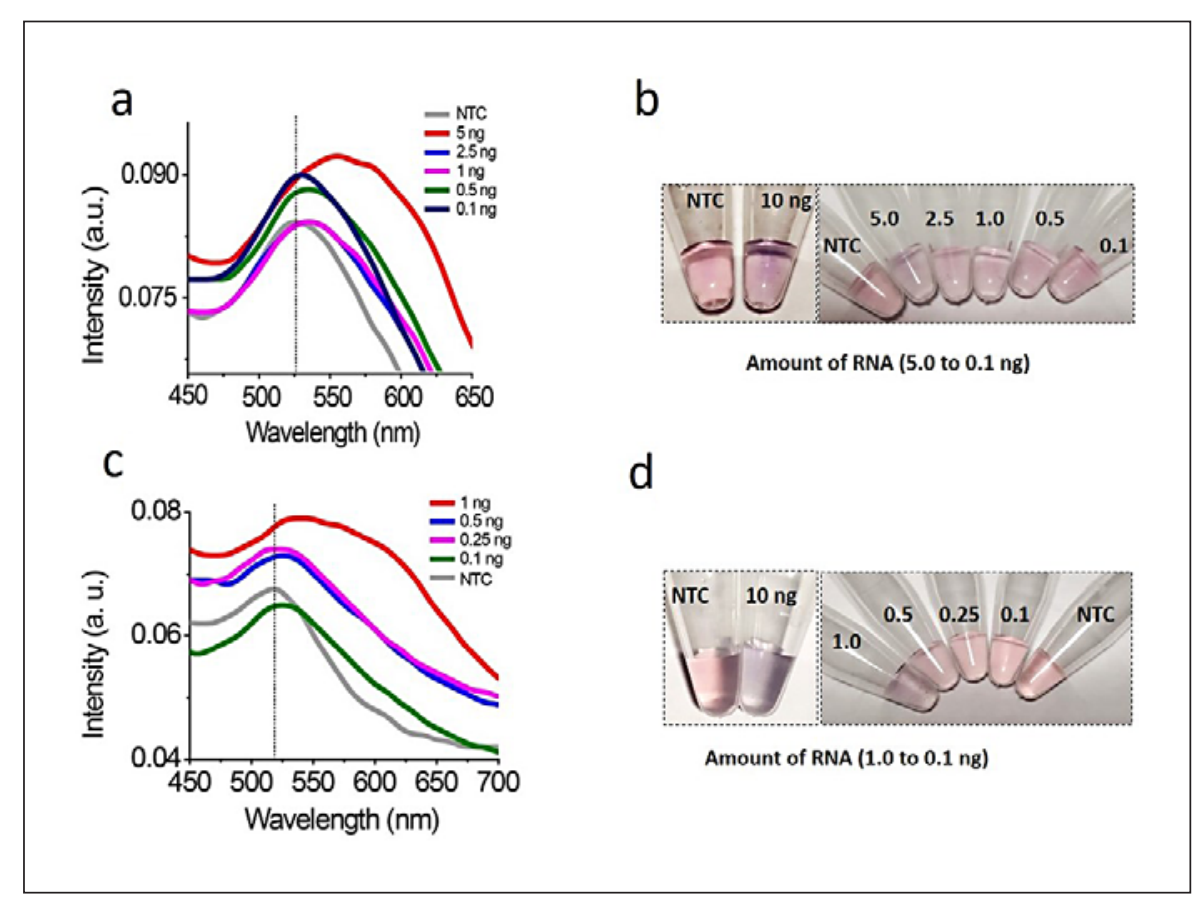

mized earlier for SARS-CoV-2 RNA and RdRp oligo probe. It established the working principle and specificity of the test (online suppl. Fig. S1).

We determined the cross-reactivity using a cervicalDNA sample from women diagnosed with HPV infection (nonspecific target control). No color change of gold colloids was observed with HPV DNA, indicating no hybridization, and specificity of the developed assay (Fig. 3b, right vial). Contrary to HPV DNA-negative control and NTC, a positive control sample shows development of blue color (Fig. 3b, middle vial). Absorption spectrum (of colloids) with HPV DNA-negative control exhibited characteristics similar to that of NTC, and no red-shift or peak broadening as found with positive samples (Fig. 3a). Cross-reactivity of the developed assay with other respiratory viruses is warranted. However, we do not anticipate the same as the test utilizes the detection of the RdRp gene of the SARS-CoV-2 virus. The oligo probe sequence used in our assay is not complementary to any human mRNAs and other members of the SARS family, as verified by BLAST using the NCBI database [10]. IVT synthesized SARS-CoV-2 RNA ranging from 0.1 to $5 \mathrm{ng}$ resulted in color difference at $0.1 \mathrm{ng}$, compared to NTC, is barely visible with naked eyes (Fig. $4 \mathrm{~b}$ extreme left vial). However, the absorption spectra of colloids show a clear red-shift with peak broadening up till $0.5 \mathrm{ng}$ target RNA (Fig. 4a).
We also determined the assay sensitivity using different dilutions (1-0.1 ng) of PCR-amplified synthetic DNA (positive control, with COVID-19 control kit). Similar to IVT synthesized RNA, a decreasing amount of DNA (from 1 to $0.1 \mathrm{ng}$ ) show a gradual change in colloid color from blue to light pink (left to right, Fig. 4d). A clear redshift with peak broadening reflects in the absorption spectra of colloids recorded with control DNA, compared to NTC (Fig. 4c). Accordingly, positive control showed a clear visual demarcation up to $0.5 \mathrm{ng}$ amount, compared to NTC (Fig. 4d). A similar recent approach utilizes thiol capped AuNPs to detect N-gene of the SARS-CoV-2 gene in a cellular system [9]. However, for the detection of SARS-CoV-2 RNA in human samples, the N-gene has reportedly inferior analytical power than the RdRp gene [10]. Thus, it would be essential to know the assay's performance, developed by Moitra et al. [10] with clinical samples. A preprint version of this article is available on BioRxiv [17].

\section{Conclusion}

We have successfully developed an affordable AuNPsbased colorimetric test for the rapid detection of SARSCoV-2 RNA in humans. The assay can detect up to $0.5 \mathrm{ng}$ of SARS-CoV-2 RNA. The turnaround time of our assay 
is less than $30 \mathrm{~min}$. Moreover, the developed test will be helpful for mass screening, as it does not require sophisticated equipment. However, while analyzing the clinical samples, we observed the assay works best with the freshly isolated RNA samples. In addition, $\mathrm{pH}$ and salt concentration in the elution buffer, used for RNA isolation, may affect the result and hence optimization may be needed when using a different kit for RNA isolation.

\section{Acknowledgment}

A patent on the subject matter has been filed (ref $\# 202011018132$ ) on subject matter described in the publication by SGPGIMS with ST, and VK as inventors. All other co-authors declare no financial and intellectual conflict of interest. Preprint version: A preprint version of this article is available on BioRxiv [17].

\section{Statement of Ethics}

The study protocol to use clinical samples was approved by Institutional Human Ethics Committee SGPGIMS, Lucknow (Ref N. PGI/BE/327/2020). For our study, we have not enrolled any participants; we received the left-over clinical samples (nasopharyngeal RNA samples) from the Department of Microbiology, SGPGIMS, and RMLIMS, Lucknow.

\section{Conflict of Interest Statement}

All other co-authors declare no financial and intellectual conflict of interest. A patent on the subject matter has been filed by SGPGIMS with S.T., and V.K. as inventors.

\section{Funding Sources}

The study was supported by intramural Grants (A-24-PGI/ IMP/81/2020) and overhead funds extramural grants supported to ST from DBT, ICMR, and MHRD. However, the funders do not have any role in designing, execution, and results of the study.

\section{Author Contributions}

S.T. and V.K.: conceived the idea; S.T., V.K., S.M., and R.S.: performed the experiments, J.Y. and U.G.: provided clinical samples and the RT-PCR diagnosis; T.K., L.K.S., P.M., and S.K.V.: provided critical comments on the manuscript draft; and S.T., V.K., and S.M.: drafted the manuscript.

\section{Data Availability Statement}

All data generated or analysed during this study are included in this article [and/or] its supplementary material files. Supplementary information supporting the finding of this study is available in this article as supporting information contains all results of patient samples. Further enquiries can be directed to the corresponding author.

\section{References}

1 https: //www.who.int/emergencies/diseases/ novel - coronavirus-2019? gclid= EAIaIQobChMI-u-Pz9vW6QIVUyUrCh0kjAIOEAAYASAAEgJ0pvD BwERemuzzi.

2 Udugama B, Kadhiresan P, Kozlowski HN, Malekjahani A, Osborne M, Li VYC, et al. Diagnosing COVID-19: the disease and tools for detection. ACS Nano. 2020;14(4):3822-35.

3 World Health Organization. Report of the WHO-China joint mission on coronavirus disease 2019 (COVID-19). Geneva: World Health Organization; 2017.

4 World Health Organization. SARS-CoV-2 antigen-detecting rapid diagnostic tests: an implementation guide. Geneva: World Health Organization; 2020. Licence: CC BYNC-SA 3.0 IGO.

5 Division of Viral Diseases. CDC 2019-novel coronavirus (2019-nCoV) real-time RT-PCR diagnostic panel; Division of Viral Diseases USCfDCaP. 2020

6 Kashir J, Yaqinuddin A. Loop mediated isothermal amplification (LAMP) assays as a rapid diagnostic for COVID-19. Med Hypotheses. 2020;141:109786.
7 Broughton JP, Deng X, Yu G, Fasching CL, Servellita V, Singh J, et al. CRISPR-Cas12based detection of SARS-CoV-2. Nature Biotechnology. 2020;38(7):870-4.

8 Qiu G, Gai Z, Tao Y, Schmitt J, Kullak-Ublick GA, Wang J. Dual-functional plasmonic photothermal biosensors for highly accurate severe acute respiratory syndrome coronavirus 2 detection. ACS Nano. 2020;14(5):5268-77.

9 Chen Z, Zhang Z, Zhai X, Li Y, Lin L, Zhao H, et al. Rapid and sensitive detection of antiSARS-CoV-2 IgG, using lanthanide-doped nanoparticles-based lateral flow immunoassay. Anal Chem. 2020;92(10):7226-31.

10 Moitra P, Alafeef M, Dighe K, Frieman MB, Pan D. Selective naked-eye detection of SARS-CoV- 2 mediated by $\mathrm{N}$ gene targeted antisense oligonucleotide capped plasmonic nanoparticles. ACS nano. 2020;14(6):617.

11 Corman VM, Landt O, Kaiser M, Molenkamp R, Meijer A, Chu DK, et al. Detection of 2019 novel coronavirus $(2019-\mathrm{nCoV})$ by real-time RT-PCR. Euro Surveill. 2020;25(3):2000045.

12 Jain PK, Lee KS, El-Sayed IH, El-Sayed MA. Calculated absorption and scattering proper- ties of gold nanoparticles of different size, shape, and composition: applications in biological imaging and biomedicine. J Phys Chem B. 2006;110(14):7238-48.

13 Lazarides AA, Schatz GC. DNA-linked metal nanosphere materials: structural basis for the optical properties. J Phys Chem B. 2000; 104(3):460-7.

14 Hunter RJFoCSOUPINY, 2001. Shaw, D. J. Colloid and Surface Chemistry; ButterworthHeinemann Ltd.: Oxford. 1991.

15 Grabar KC, Freeman RG, Hommer MB, Na$\tan$ MJ. Preparation and characterization of Au colloid monolayers. Anal Chem. 1995; 67(4):735-43.

$16 \mathrm{Li} \mathrm{H}$, Rothberg L. Colorimetric detection of DNA sequences based on electrostatic interactions with unmodified gold nanoparticles. Proc Natl Acad Sci U S A. 2004;101(39): 14036-9.

17 Kumar V, Mishra S, Sharma R, Agarwal J, Ghoshal U, Khanna T, et al. Development of RNA-based assay for rapid detection of SARS-CoV-2 in clinical samples. bioRxiv. 2020;0630:172833. 